\title{
Full-thickness resection with an over-the-scope device: possible translocation of adenoma tissue in a case of an incomplete resection at the appendix
}

\section{(ㄷ)(1) $\odot(9$}

\author{
Authors \\ Institutions \\ 1 Department of Medicine and Gastroenterology, \\ Gemeinschaftskrankenhaus Bonn, Bonn, Germany \\ 2 Office for Internal Medicine, Cardiology and \\ Gastroenterology, St. Augustin, Germany \\ 3 Institute für Pathology Bonn-Duisdorf, Bonn, Germany \\ 4 Department of General and Abdominal Surgery, \\ Gemeinschaftskrankenhaus Bonn, Bonn, Germany
}

Franz Ludwig Dumoulin' ${ }^{1}$ Dominik G. Gorris ${ }^{1}$, Susanne Berger ${ }^{2}$, Ralf Hildenbrand ${ }^{3}$, Bernd Sido ${ }^{4}$

submitted 3.11.2017

accepted after revision 12.2 .2018

\author{
Bibliography \\ DOI https://doi.org/10.1055/a-0589-9850 | \\ Endoscopy International Open 2018; 06: E622-E624 \\ (c) Georg Thieme Verlag KG Stuttgart · New York \\ ISSN 2364-3722
}

Corresponding author

Prof. Dr. Franz Ludwig Dumoulin, Department of Medicine and Gastroenterology, Gemeinschaftskrankenhaus Bonn,
Bonner Talweg 4-6, D-53113, Bonn, Germany

Fax: +49-228-508-1562

f.dumoulin@gk-bonn.de

d.gorris@gk-bonn.de

\section{ABSTRACT}

Background and study aims Clip-assisted endoscopic full-thickness resection has great potential for treatment of difficult-to-resect colorectal neoplasia. Here, we report on endoscopic full-thickness resection at the appendiceal orifice with the appendix in situ requiring emergency surgery due to acute appendicitis. Final histopathology showed appendicitis and residual serrated adenoma within the appendiceal stump, but unexpectedly, also a displaced adenoma fragment at the serosal surface of the cecum. Given the transmural placement of the clip prior to snare resection, translocation of neoplastic tissue to the extra luminal site in cases of incomplete adenoma/carcinoma resection could be a concern.

\section{Introduction}

The endoscopic full-thickness resection device (FTRD, Ovesco, Germany) combines a modified over-the-scope-clip (OTSC) for creation of a full thickness plication and a snare, which is subsequently used to cut a full-thickness specimen [1,2]. The method has great potential, particularly for treatment of difficult-toresect colorectal neoplasia such as non-lifting lesions (e.g. due to scarring after previous endoscopic treatment) or lesions in specific anatomic localizations (e. g. in proximity to a diverticulum or at the appendiceal orifice) [3-5]. The largest study published so far included 181 patients and reported R0 resection rates of $77.7 \%$ and $72.4 \%$ for difficult-to-resect colorectal adenomas and early cancers, respectively. The rate of emergency surgery was $2 \%$ and included one case of acute appendicitis and an enterocolonic fistula after EFTR at the appendix [6]. Given transmural placement of the modified OTSC clip, translocation of neoplastic tissue to the extra luminal site in cases of incomplete adenoma/carcinoma resection is a concern. Here, we present a case of possible translocation of a neoplastic tissue fragment during incomplete adenoma resection at the appendiceal orifice.

\section{Case report}

A 65-year-old woman was referred for endoscopic treatment of a serrated adenoma surrounding the appendiceal orifice with the appendix in situ. The resection was performed with a colonoscope (CF-H180AL, Olympus Medical, Germany) using the FTRD system without any technical problems. The resected specimen was apparently complete but histopathology revealed residual neoplastic tissue at the margin towards the appendiceal stump ( $\triangleright$ Fig.1). Moreover, and despite antibiotic prophylaxis and therapy (initial prophylaxis with cefuroxime continued after the intervention with ceftriaxone/metronidazole), the patient developed acute appendicitis. Within 6 hours after the intervention, she reported nausea, vomiting and abdominal discomfort. Her symptoms gradually increased in se- 

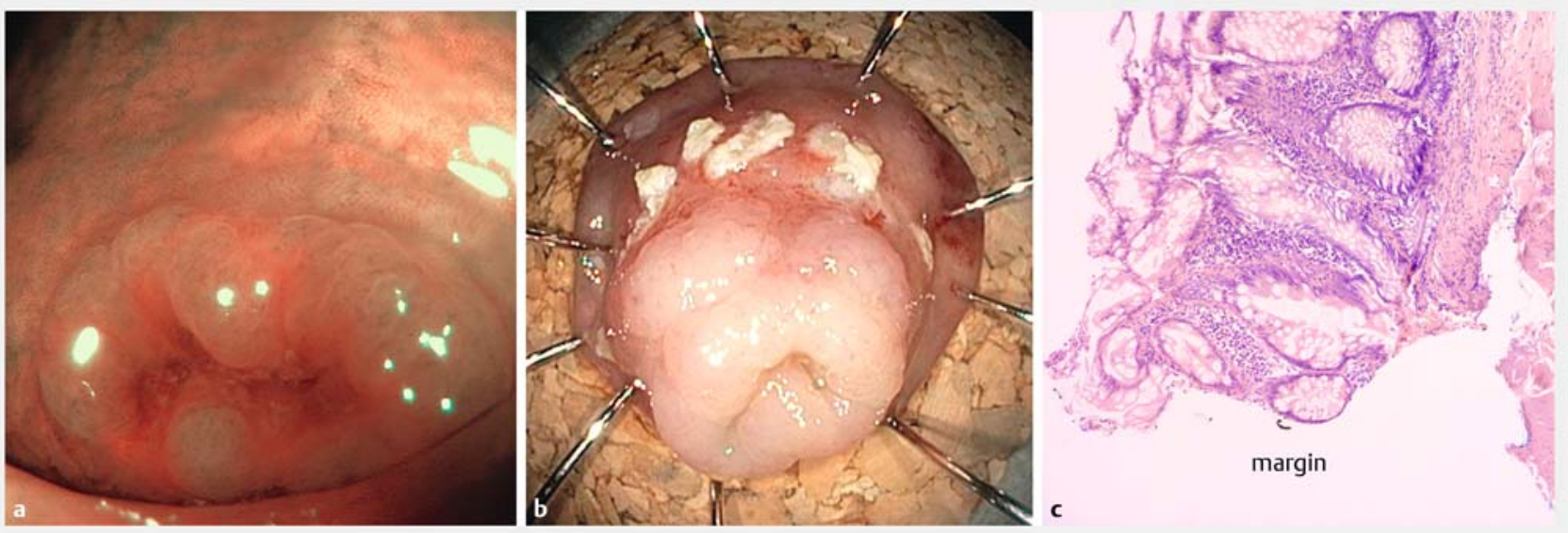

- Fig. 1 Endoscopic full-thickness resection of a serrated adenoma at the appendiceal orifice. Left: Narrow band imaging of the serrated adenoma, a neoplastic 0 -Ila lesions. Center: Resected specimen with apparently complete resection. Right: Histopathology of the resected specimen with a positive margin.
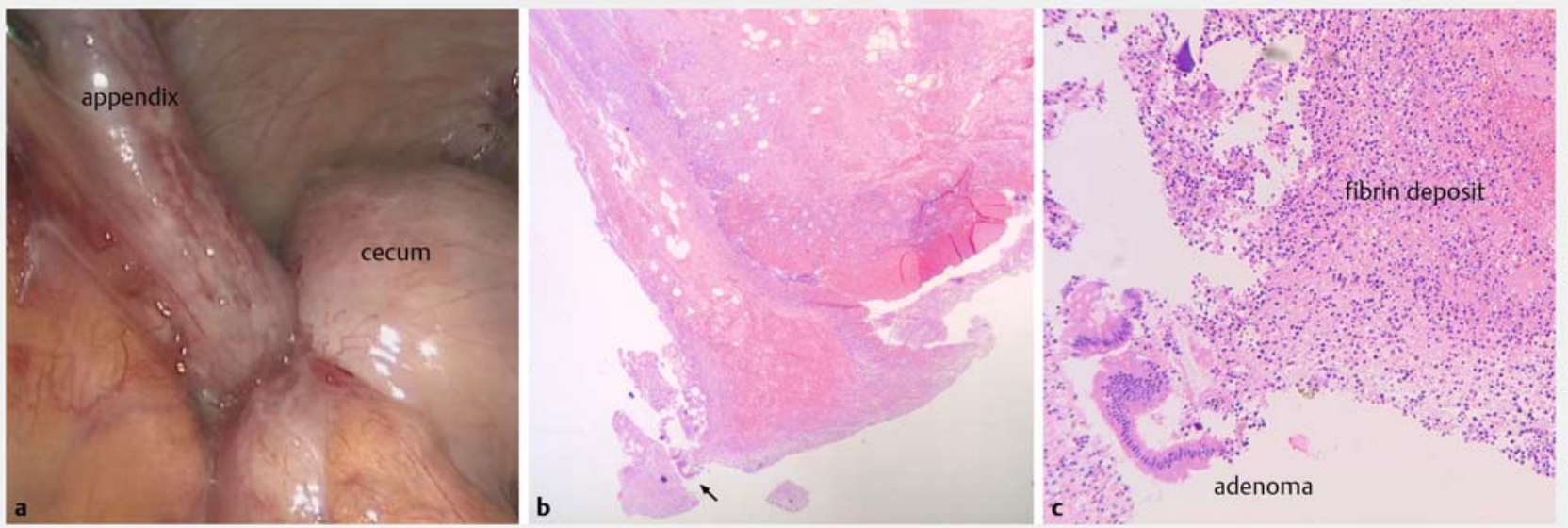

- Fig.2 Surgical resection after development of acute appendicitis. Left: Laparoscopic appearance of the appendix with typical signs of acute appendicitis. Center: Low magnification of resected specimen with small adenoma fragment (black arrow). Right: Small adenoma fragment embedded in fibrin deposit at the serosal surface of the resected specimen.

verity with fever, increasing leucocyte counts and typical signs of appendicitis on transabdominal ultrasound. On the second post-interventional day, laparoscopic ileocecal resection (rather than the planned cecal pole resection) had to be performed due to the close proximity of the OTSC clip to the ileocecal valve. The final histopathology not only showed acute appendicitis and residual serrated adenoma within the appendiceal stump but also displaced adenoma fragment attached to serosal surface of the cecum ( $\mathbf{F i g . 2}$ ).

\section{Discussion}

Here we report the unfortunate course after EFTR at the appendiceal orifice with the appendix in situ. Despite prophylactic and therapeutic use of antibiotics, the patient developed signs of acute appendicitis with need for emergency surgery. Appen- dicitis after EFTR necessitating surgery at this location has been described [6] and it is most probably the consequence of luminal obstruction [7]. Of note, in this particular case, a laparoscopic ileocecal resection had to be performed (rather than a simple cecal pole resection, which would have been the alternative to endoscopic treatment).

When analyzing the resected specimen, we observed a neoplastic tissue fragment embedded in the fibrinous deposits on the outer (serosal) surface or the cecum. While it is impossible to definitely prove that the neoplastic tissue fragment was displaced during the EFTR procedure (rather than during emergency surgery or processing of the specimen) the circumstantial evidence argues in favor of such an event. In fact, inadvertent placement of the modified OTSC clip through neoplastic tissue represents at least a theoretical risk of tumor cell dissemination to the extra luminal side of the colon. Tumor cell 
spread after peritoneal perforation has been reported for endoscopic submucosal dissection of early gastric cancer [8] or after complicated transanal endoscopic microsurgery [9]. It is probably a rare event and its clinical significance is unclear, in particular since data on long-term follow-up are lacking. Nevertheless, incomplete resections mostly resulting from a lack of control of the lateral resection margins have been reported in roughly every fourth EFTR intervention [6]. Therefore, longterm follow-up data will be important to rule out that possible spread of neoplastic tissue might have clinical consequences. Given the data presented above, an $\mathrm{R} 1$ resection of invasive cancer is suggestive of clip placement through the cancer and consequently, additional surgery should be considered.

\section{Conclusion}

In summary, we recommend avoiding EFTR with the FTRD system for neoplastic lesions surrounding the appendiceal orifice when the appendix is in situ because of a higher risk of incomplete resection, possible translocation of neoplastic tissue and risk of appendicitis. Instead, laparoscopic cecal pole resection should be the preferred method. Moreover, every possible measure should be undertaken to avoid placement of the clip through neoplastic tissue.

Competing interests

None

\section{References}

[1] Schurr MO, Buess G, Raestrup H et al. Full thickness resection device (FTRD) for endoluminal removal of large bowel tumours: development of the instrument and related experimental studies. MITAT 2009; 10: $301-309$

[2] Schurr MO, Baur FE, Krautwald M et al. Endoscopic full-thickness resection and clip defect closure in the colon with the new FTRD system: experimental study. Surg Endosc 2015; 29: 2434-2441

[3] Sarker S, Gutierrez JP, Council L et al. Over-the-scope clip-assisted method for resection of full-thickness submucosal lesions of the gastrointestinal tract. Endoscopy 2014; 46: 758 - 761

[4] Fahndrich M, Sandmann M. Endoscopic full-thickness resection for gastrointestinal lesions using the over-the-scope clip system: a case series. Endoscopy 2015; 47: $76-79$

[5] Al-Bawardy B, Rajan E, Wong KeeSong LM. Over-the-scope clip-assisted endoscopic full-thickness resection of epithelial and subepithelial Gl lesions. Gastrointest Endosc 2017; 85: 1087-1092

[6] Schmidt A, Beyna T, Schumacher B et al. Colonoscopic full-thickness resection using an over-the-scope device: a prospective multicentre study in various indications. Gut 2017: doi:10.1136/gutjnl-2016313677 [Epub ahead of print Aug 10]

[7] Baird DLH, Simillis C, Kontovounisios C et al. Acute appendicitis. BM] (Clinical research ed) 2017; 357: j1703

[8] Hirao M, Yamada T, Michida T et al. Peritoneal seeding after gastric perforation during endoscopic submucosal dissection for gastric cancer. Dig Surg 2017: doi:10.1159/000481715 [Epub ahead of print Nov 8]

[9] Mege D, Petrucciani N, Maggiori L et al. Peritoneal perforation is less a complication than an expected event during transanal endoscopic microsurgery: experience from 194 consecutive cases. Tech Coloproctol 2017; 21: 729-736 